CLINICAL STUDY

\title{
Chemerin is associated with markers of inflammation and components of the metabolic syndrome but does not predict coronary atherosclerosis
}

\author{
Michael Lehrke ${ }^{1}$, Alexander Becker ${ }^{2}$, Martin Greif $^{2}$, Renee Stark ${ }^{3}$, Rüdiger P Laubender ${ }^{4}$, Franz von Ziegler ${ }^{2}$, \\ Corinna Lebherz ${ }^{2}$, Janine Tittus ${ }^{2}$, Maximilian Reiser ${ }^{5}$, Christoph Becker ${ }^{5}$, Burkhard Göke ${ }^{1}$, Alexander W Leber ${ }^{2}$, \\ Klaus G Parhofer ${ }^{1}$ and Uli C Broedl ${ }^{1}$ \\ Departments of ${ }^{1}$ Internal Medicine II and ${ }^{2}$ Internal Medicine I, University of Munich, Campus Grosshadern, Marchioninistrasse 15, 81377 Munich, \\ Germany, ${ }^{3}$ Helmholtz-Zentrum, Munich, Germany, ${ }^{4}$ Institute for Medical Informatics, Biometry and Epidemiology (IBE) and ${ }^{5}$ Department of Radiology, \\ University of Munich, Munich, Germany \\ (Correspondence should be addressed to U C Broedl; Email: uli.broedl@med.uni-muenchen.de)
}

\begin{abstract}
Objectives: Chemerin is a recently discovered adipokine that regulates adipocyte differentiation and modulates chemotaxis and activation of dendritic cells and macrophages. Given the convergence of adipocyte and macrophage function, chemerin may provide an interesting link between obesity, inflammation and atherosclerosis in humans. We sought to examine the relationship of i) chemerin and markers of inflammation, ii) chemerin and components of the metabolic syndrome, and iii) chemerin and coronary atherosclerotic plaque burden and morphology.

Design: Serum chemerin levels were determined in 303 patients with stable typical or atypical chest pain who underwent dual-source multi-slice CT-angiography to exclude coronary artery stenosis. Atherosclerotic plaques were classified as calcified, mixed, or non-calcified.

Results: Chemerin levels were highly correlated with high sensitivity C-reactive protein $(r=0.44$, $P<0.0001)$, interleukin-6 $(r=0.18, P=0.002)$, tumor necrosis factor $\alpha(r=0.24, P<0.0001)$, resistin $(r=0.28, P<0.0001)$, and leptin $(r=0.36, P<0.0001)$ concentrations. Furthermore, chemerin was associated with components of the metabolic syndrome including body mass index $(r=0.23, P=0.0002)$, triglycerides $(r=0.29, P<0.0001)$, HDL-cholesterol $(r=-0.18, P=0.003)$, and hypertension $(P<0.0001)$. In bivariate analysis, chemerin levels were weakly correlated with coronary plaque burden $(r=0.16, P=0.006)$ and the number of non-calcified plaques $(r=0.14$, $P=0.02)$. These associations, however, were lost after adjusting for established cardiovascular risk factors (odds ratio, OR 1.17, 95\% confidence interval (CI) $0.97-1.41, P=0.11$ for coronary plaque burden; OR 1.06, 95\% CI 0.96-1.17, $P=0.22$ for non-calcified plaques).

Conclusions: Chemerin is strongly associated with markers of inflammation and components of the metabolic syndrome. However, chemerin does not predict coronary atherosclerosis.
\end{abstract}

European Journal of Endocrinology 161 339-344

\section{Introduction}

Obesity and atherosclerosis are increasingly viewed as inflammatory states. Biomarkers that integrate metabolic and inflammatory signals may be attractive candidates for assessing risk of atherosclerotic cardiovascular disease (1). Chemerin is a recently discovered chemokine (2) highly expressed in liver and white adipose tissue $(3,4)$ that modulates chemotaxis and activation of dendritic cells and macrophages through distinct $\mathrm{G}$ protein-coupled receptors such as CMKLR1, GPR1, and CCRL2 (5-7). Upon secretion, enzymatic proteolysis of pro-chemerin can result in both activating and inhibitory peptides. These molecules with opposing activities are generated by different classes of proteases (8). Serine proteases released by granulocytes upon degranulation were shown to cleave the C-terminal extremity of pro-chemerin and to release its chemotactic potential (9). Cysteine proteases derived from activated macrophages cleave chemerin to generate potent antiinflammatory products that inhibit the production of pro-inflammatory mediators such as tumor necrosis factor- $\alpha$ (TNF- $\alpha$ ), interleukin-1 $\beta$ (IL-1 $\beta$ ), and IL- 6 while inducing the expression of anti-inflammatory cytokines such as transforming growth factor- $\beta$ and IL-10 (5). In addition to its immunomodulatory effects, chemerin was reported to be associated with components of the metabolic syndrome including body mass index (BMI), plasma triglyceride (TG) levels, and hypertension (3). Furthermore, chemerin was shown to regulate adipocyte differentiation in an autocrine/paracrine manner via the CMKLR1 receptor, to modulate the expression of 
adipocyte genes involved in glucose and lipid homeostasis such as glucose transporter- 4 , fatty acid synthase, adiponectin, and leptin (4), and to enhance insulin signaling in 3T3-L1 adipocytes (10). Given the convergence of adipocyte and macrophage function, chemerin may provide an interesting link between obesity, inflammation, and atherosclerosis in humans. To examine the relationship of chemerin and markers of inflammation, components of the metabolic syndrome, and coronary atherosclerosis, we determined serum chemerin levels in 303 patients with stable typical or atypical chest pain who underwent dual-source CT (DSCT)-coronary angiography to exclude coronary artery stenosis.

\section{Materials and methods}

\section{Ascertainment of subjects}

A total of 303 consecutive (relating to the order in which they attended the clinic) patients who underwent DSCT-coronary angiography for exclusion of coronary artery stenosis due to stable typical or atypical chest pain were recruited during 20 consecutive months from March 2006 to October 2007 as previously described (11). All patients were of Caucasian origin. After providing informed written consent, study subjects were asked to complete a brief questionnaire and have blood drawn. The study protocol was approved by the Ethics Committee of the LudwigMaximilians-University Munich, Germany. Baseline characteristics of the study cohort (Table 1) have in part been previously reported (11).

\section{Dual-source multi-slice CT-coronary angiography}

CT-coronary angiography and image analyses were performed as previously described $(11,12)$. Briefly, for the analysis of coronary plaque morphology, all reconstructed data sets were evaluated at different ECG-phases for diagnostic image quality and the optimal data set was then chosen. The DSCT datasets were evaluated by two independent investigators blinded to serum chemerin levels using a dedicated cardiac workstation (Siemens, Leonardo Circulation). Atherosclerotic plaques were classified as calcified, mixed, or non-calcified. Calcified plaques were defined as lesions with a HU value above 130 . Non-calcified plaques were defined as structures clearly assignable to the vessel wall (in at least two views) with densities less than the lumen contrast. Plaques with $<50 \%$ calcified plaque area were classified as mixed. The coronary tree was segmented according to the suggestions of the American Heart Association (AHA) into a 15 segment model. Each segment was further divided into a proximal and a distal segment. Each segment was then classified as containing either calcified, noncalcified, mixed, or no plaque. Based on the number of diseased segments, a plaque score was calculated. Adequate image quality for evaluation of coronary plaques was obtained in 281 out of 303 patients.

The same images as for the analysis of atherosclerotic plaques were used to determine pericardial adipose tissue (PAT) volume. PAT volume was measured in milliliters using the volume analysis software tool of the Siemens Leonardo Circulation workstation. PAT volume was determined as previously described $(11,12)$. We defined PAT as the adipose tissue surrounding the myocardium. The upper cut-off point in the axial slices was the bifurcation of the pulmonary artery. Adequate image quality for evaluation of PAT volume was obtained in 287 out of 303 patients.

\section{Laboratory procedures}

Blood samples were stored at $-70{ }^{\circ} \mathrm{C}$ until analysis. Serum levels of adiponectin, chemerin, leptin, and resistin were determined with commercially available ELISAs (R\&D, Wiesbaden, Germany). Both, the monoclonal capture and the polyclonal detection antibody used for the chemerin ELISA were raised against residues 21-157 of recombinant human chemerin. Therefore, the assay used in this study measures prochemerin, chemerin, and likely some of the proteolytically processed short forms. Unfortunately, there is no information available regarding the affinity of these antibodies to the different chemerin forms. Intra- and inter-assay coefficients of variance for the chemerin ELISA were 2.7 and $9.3 \%$ respectively.

Plasma LDL-cholesterol (LDL-C), HDL-cholesterol (HDL-C), and TG were measured by routine enzymatic methods. Determination of high sensitivity C-reactive protein (hsCRP), IL- 6 , and TNF- $\alpha$ levels was performed at the Department of Clinical Chemistry (Campus Grosshadern, University of Munich, Germany).

\section{Statistical analysis}

Statistical analyses were performed by $\mathrm{R}$ 2.8.1 (Vienna, Austria) software. Data are reported as $n$ or median (interquartile range). Spearman correlation and Wilcoxon two-sample test were used in the bivariate analysis of chemerin with other variables. A generalized linear regression model was used to assess the association of chemerin serum levels with atherosclerotic plaque burden, number of calcified plaques, mixed plaques, or non-calcified plaques adjusted for age, sex, BMI, diabetes, hypertension, family history of coronary artery disease (CAD), smoking, LDL-C, HDL-C, TG, hsCRP, IL- 6 , TNF- $\alpha$, leptin, resistin, adiponectin, PAT volume, and medication - possible confounders of chemerin levels. All tests were two-tailed with a 0.05 type I error rate. 
Table 1 Baseline characteristics and CT-angiographic findings of the study population.

\begin{tabular}{|c|c|}
\hline \multicolumn{2}{|l|}{ Characteristics } \\
\hline Age (years) & $63(55-70)$ \\
\hline $\operatorname{Sex}(m / f)$ & $202 / 101$ \\
\hline Body mass index $\left(\mathrm{kg} / \mathrm{m}^{2}\right)$ & $26.2(24.1-29.0)$ \\
\hline Hypertension $^{\mathrm{a}}$ & $54 \%$ \\
\hline Diabetes mellitus $^{a}$ & $7 \%$ \\
\hline Smoker $^{\mathrm{b}}$ & $15 \%$ \\
\hline Family history of $C A D^{b}$ & $28 \%$ \\
\hline \multicolumn{2}{|l|}{ Laboratory profile } \\
\hline LDL-cholesterol (mg/dl) & $122(95-149)$ \\
\hline HDL-cholesterol (mg/dl) & $52(44-59)$ \\
\hline Triglycerides (mg/dl) & $143(106-207)$ \\
\hline High-sensitivity CRP (mg/dl) & $0.23(0.05-0.54)$ \\
\hline $\mathrm{IL}-6(\mathrm{pg} / \mathrm{ml})^{\mathrm{c}}$ & $2.0(2.0-3.16)$ \\
\hline TNF- $\alpha(\mathrm{pg} / \mathrm{ml})$ & $6.3(4.5-7.9)$ \\
\hline Resistin (ng/ml) & $11.5(9.4-14.3)$ \\
\hline Leptin (ng/ml) & $6.9(3.6-16.1)$ \\
\hline Adiponectin $(\mu \mathrm{g} / \mathrm{ml})$ & $5.1(3.3-7.8)$ \\
\hline Chemerin $(\mathrm{ng} / \mathrm{ml})$ & $108(88-133)$ \\
\hline \multicolumn{2}{|l|}{ Medical treatment ${ }^{d}$} \\
\hline Statin & 120 \\
\hline Acetylsalicylic acid, clopidogrel or marcumar & 170 \\
\hline Betablocker & 173 \\
\hline ACE-I or ARB & 138 \\
\hline Diuretics & 87 \\
\hline Insulin or OAD & 16 \\
\hline \multicolumn{2}{|l|}{ CT data } \\
\hline Pericardial adipose tissue volume $(\mathrm{ml})^{\mathrm{e}}$ & $190(132-259)$ \\
\hline Number of coronary artery plaques (total) ${ }^{f}$ & $3(1-6)$ (range $0-26)$ \\
\hline Number of calcified plaques (154 patients) & 1 (0-3) (range 0-22) \\
\hline Number of mixed plaques (102 patients) & $0(0-1)$ (range $0-10)$ \\
\hline Number of non-calcified plaques (126 patients) & $0(0-2)$ (range $0-9)$ \\
\hline
\end{tabular}

Values are presented as $n$ or median (interquartile range).

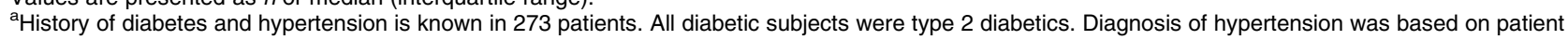
history.

${ }^{b}$ History of smoking and family history of CAD are known in 272 patients.

'Detection limit of the IL-6 assay was $2 \mathrm{pg} / \mathrm{ml}$.

${ }^{\mathrm{d}}$ Medication is known in 258 patients.

${ }^{\text {e}}$ Adequate image quality for evaluation of PAT volume was obtained in 287 patients.

${ }^{\mathrm{f}}$ Adequate image quality for evaluation of coronary plaques was obtained in 281 patients. A total of 60 patients had no coronary plaques (21\%).

\section{Results}

\section{Chemerin is associated with markers of inflammation and components of the meta- bolic syndrome}

Chemerin was strongly correlated with all markers of inflammation including hsCRP $(r=0.44, P<0.0001)$, IL-6 $\quad(r=0.18, \quad P=0.002), \quad$ TNF- $\alpha \quad(r=0.24$, $P<0.0001)$, resistin $(r=0.28, P<0.0001)$, and leptin $(r=0.36, P<0.0001$; Table 2$)$. The association of chemerin with hsCRP persisted after adjusting for anti-inflammatory drugs such as acetylsalicylic acid and statins $(P<0.0001)$.

Chemerin was positively correlated with BMI $(r=0.23, P=0.0002)$ and PAT volume $(r=0.20$, $P=0.0007)$ defined as the volume of adipose tissue surrounding the myocardium with an upper cut-off point determined by the bifurcation of the pulmonary artery, and TG $(r=0.29, P<0.0001)$, yet negatively correlated with HDL-C $(r=-0.18, \quad P=0.003)$.
The association of chemerin with TG remained significant after adjusting for BMI $(P<0.0001)$ or lipid lowering therapy $(P<0.0001)$. Chemerin did not correlate with LDL-C $(r=0.05, P=0.43)$ and adiponectin $(r=-0.09, P=0.15)$.

Among demographic characteristics, hypertension $(P<0.0001)$ was associated with higher chemerin levels. This association persisted after adjusting for anti-hypertensive agents $(P=0.009)$. No association was seen with diabetes mellitus $(P=0.15)$, smoking $(P=0.23)$, and family history of $\mathrm{CAD}(P=0.96)$.

\section{Chemerin does not predict coronary athero- sclerotic burden or plaque morphology}

In bivariate analysis, chemerin levels weakly correlated with coronary plaque burden $(r=0.16, P=0.006)$ and the number of non-calcified plaques $(r=0.14, P=0.02$; Table 2). After adjusting for established cardiovascular risk factors, these associations were lost (odds ratio, 
Table 2 Correlations and associations between chemerin and makers of inflammation, components of the metabolic syndrome, and coronary atherosclerosis.

\begin{tabular}{|c|c|c|}
\hline \multicolumn{2}{|l|}{ Characteristics } & \multirow{3}{*}{$\begin{array}{c}\boldsymbol{P} \\
0.0002 \\
0.0003\end{array}$} \\
\hline Age & 0.22 & \\
\hline Sex & & \\
\hline Male & $102(84-126)$ & \\
\hline Female & $121(95-140)$ & \\
\hline Body mass index $\left(\mathrm{kg} / \mathrm{m}^{2}\right)$ & 0.23 & 0.0002 \\
\hline Actual hypertension & & $<0.0001$ \\
\hline Yes & $122(99-148)$ & \\
\hline No & $100(81-120)$ & \\
\hline Diabetes mellitus & & 0.15 \\
\hline Yes & $127(90-145)$ & \\
\hline No & $108(89-132)$ & \\
\hline Actual smoker & & 0.23 \\
\hline Yes & $109(93-144)$ & \\
\hline No & $108(88-132)$ & \\
\hline Family history of CAD & & 0.96 \\
\hline Yes & $111(90-133)$ & \\
\hline No & $109(88-134)$ & \\
\hline LDL-cholesterol (mg/dl) & 0.05 & 0.43 \\
\hline HDL-cholesterol (mg/dl) & -0.18 & 0.003 \\
\hline Triglycerides $(\mathrm{mg} / \mathrm{dl})$ & 0.29 & $<0.0001$ \\
\hline High-sensitivity ČRP (mg/dl) & 0.44 & $<0.0001$ \\
\hline $\mathrm{IL}-6(\mathrm{pg} / \mathrm{ml})$ & 0.18 & 0.002 \\
\hline TNF- $\alpha(\mathrm{pg} / \mathrm{ml})$ & 0.24 & $<0.0001$ \\
\hline Resistin (ng/ml) & 0.28 & $<0.0001$ \\
\hline Leptin $(\mathrm{ng} / \mathrm{ml})$ & 0.36 & $<0.0001$ \\
\hline Adiponectin $(\mu \mathrm{g} / \mathrm{ml})$ & -0.09 & 0.15 \\
\hline Pericardial adipose tissue (ml) & 0.20 & 0.0007 \\
\hline $\begin{array}{l}\text { Total number of } \\
\text { coronary artery plaques }\end{array}$ & 0.16 & 0.006 \\
\hline Number of calcified plaques & 0.11 & 0.06 \\
\hline Number of mixed plaques & 0.05 & 0.37 \\
\hline Number of non-calcified plaques & 0.14 & 0.02 \\
\hline
\end{tabular}

Values are presented as correlation coefficient or median (interquartile range).

OR $1.17,95 \%$ CI $0.97-1.41, P=0.11$ for coronary plaque burden (Table 3); OR 1.06, 95\% CI 0.96-1.17, $P=0.22$ for non-calcified plaques). Further adjustment for markers of inflammation, PAT volume and medication did not alter the results.
No association was seen between chemerin and calcified, or mixed plaques in either bivariate (Table 2) or multivariate analyses (data not shown).

\section{Discussion}

Our study was aimed to address the relationship of chemerin and inflammation, components of the metabolic syndrome and atherosclerosis. Increased levels of TNF- $\alpha$, IL- 6 , CRP, leptin, and resistin have been linked to obesity and atherosclerotic cardiovascular disease (13-18). In the present study, we found chemerin to strongly correlate with all of these markers of inflammation. Furthermore, chemerin was correlated with BMI, ectopic adipose tissue (PAT), TG, low HDL-C and hypertension, components of the metabolic syndrome trait cluster. Considering the convergence of inflammation and the metabolic syndrome in the pathogenesis of atherosclerotic cardiovascular disease, we expected chemerin to correlate with coronary atherosclerosis. However, in a multivariate model adjusted for established cardiovascular risk factors chemerin was not associated with coronary atherosclerotic plaque burden or morphology.

Our study has several strengths and limitations. To our knowledge, this is the first study to examine the association of chemerin and systemic markers of inflammation as well as atherosclerotic plaque burden and morphology in a well characterized cohort of subjects with stable chest pain. Furthermore, our study supports previous data (3) demonstrating that chemerin is associated with components of the metabolic syndrome.

Chemerin was shown to have both pro- and antiinflammatory properties depending on the modality of enzymatic cleavage by different classes of proteases (8). Our study cannot address whether positive correlations of chemerin with several markers of inflammation

Table 3 Multivariate association of chemerin levels with coronary atherosclerotic plaque burden.

\begin{tabular}{lccc}
\hline & \multicolumn{3}{c}{ Coronary atherosclerotic plaque burden } \\
\cline { 2 - 4 } & OR & $95 \% \mathrm{Cl}$ & $P$ \\
\hline Model 1 & & $1.06-1.48$ & 0.008 \\
Model 2 $^{\mathrm{b}}$ & 1.25 & $0.97-1.41$ & 0.11 \\
Model 3 $_{\text {Model 4 }}^{\mathrm{d}}$ & 1.17 & $0.98-1.49$ & 0.08 \\
Model 5 $^{\mathrm{e}}$ & 1.21 & $0.95-1.49$ & 0.14 \\
Model 6 $^{\mathrm{d}}$ & 1.19 & $0.85-1.38$ & 0.53 \\
\hline
\end{tabular}

Odds ratio $(\mathrm{OR})$ and corresponding $95 \% \mathrm{Cl}$ for the association of having coronary atherosclerotic plaques for a $10 \mathrm{ng} / \mathrm{ml}$ increase in serum chemerin levels.

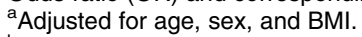

${ }^{\mathrm{b}}$ Adjusted for age, sex, BMI, hypertension, diabetes mellitus, smoking, family history of CAD, LDL-C, HDL-C, and TG.

${ }^{\mathrm{c}}$ Adjusted for age, sex, BMI, hypertension, diabetes mellitus, smoking, family history of CAD, LDL-C, HDL-C, TG, and hsCRP levels.

${ }^{\text {d} A d j u s t e d ~ f o r ~ a g e, ~ s e x, ~ B M I, ~ h y p e r t e n s i o n, ~ d i a b e t e s ~ m e l l i t u s, ~ s m o k i n g, ~ f a m i l y ~ h i s t o r y ~ o f ~ C A D, ~ L D L-C, ~ H D L-C, ~ T G, ~ h s C R P, ~ I L-6, ~ T N F-~} \alpha$, leptin, resistin, and adiponectin levels.

${ }^{\text {e} A d j u s t e d ~ f o r ~ a g e, ~ s e x, ~ B M I, ~ h y p e r t e n s i o n, ~ d i a b e t e s ~ m e l l i t u s, ~ s m o k i n g, ~ f a m i l y ~ h i s t o r y ~ o f ~ C A D, ~ L D L-C, ~ H D L-C, ~ T G, ~ h s C R P, ~ I L-6, ~ T N F-~} \alpha$, leptin, resistin, adiponectin levels, and PAT volume.

${ }^{f}$ Adjusted for age, sex, BMI, hypertension, diabetes mellitus, smoking, family history of CAD, LDL-C, HDL-C, TG, hsCRP, IL-6, TNF- $\alpha$, leptin, resistin, adiponectin levels, PAT volume, and medication. 
indicate a pro-inflammatory role of chemerin in patients with known cardiovascular risk factors and/or coronary artery disease. It is also conceivable that chemerin may be up-regulated in states of inflammation such as obesity, metabolic syndrome, and atherosclerosis to dampen inflammatory processes and to improve metabolic regulation. Assessment of chemerin cleavage products will be crucial to better define the role of chemerin in vivo. Unfortunately, the chemerin ELISA used in the present study measures prochemerin, chemerin, and likely some of the proteolytically processed short forms. Since the full-length isoform of chemerin was reported to have significantly lower bioactivity compared with its proteolytic peptides (8) the overall significance for total chemerin assessment is limited. Lack of association between total chemerin and coronary atherosclerosis in our cross-sectional study suggests that total chemerin levels do not reflect atherosclerotic plaque burden. However, it does not rule out the possibility that distinct chemerin forms may play a role in the pathogenesis of atherosclerosis. Insufficient statistical power may be another potential explanation for the lack of association between total chemerin and coronary atherosclerosis. Experiments in animal models of atherosclerosis are needed to examine the impact of chemerin on atherogenesis.

Our study population comprised patients with stable typical or atypical chest pain. It remains to be determined whether chemerin levels may be associated with acute coronary syndrome, e.g. as a marker of plaque rupture and thrombosis.

Chemerin was reported to improve insulin sensitivity in 3T3-L1 adipocytes in vitro (10). However, in humans no association of chemerin and measures of insulin resistance or diabetes were found in the only study examining this issue so far (3). Unfortunately, in the present study, parameters of insulin resistance were not available in the non-diabetic subjects, and diabetic patients were underrepresented in our cohort $(7 \%$ of patients). Therefore, our study cannot address the association of chemerin and insulin resistance or diabetes. Furthermore, the association of chemerin and atherosclerosis in insulin resistant and/or diabetic subjects remains to be determined.

Goralski et al. (4) reported that transduction of adipocytes with adenoviral vectors expressing shRNA for chemerin resulted in reduced adiponectin secretion into adipocyte media. Given the effect of chemerin on adiponectin expression in vitro and the association of adiponectin (11) and chemerin with BMI and PAT volume in vivo, we expected chemerin to be inversely correlated with serum adiponectin levels. The lack of association may be due to the determination of total chemerin rather than specific chemerin forms by our ELISA. Furthermore, assessment of high molecular weight adiponectin rather than total adiponectin may be more appropriate to examine the association of chemerin and adiponectin.
Our study population consisted of Caucasian subjects. Therefore, findings may not be generalizable to other ethnicities.

Finally, we cannot rule out the possibility that recruitment of 303 consecutive subjects (relating to the order in which they attended the clinic) may have resulted in an ascertainment bias due to selection of subjects living in proximity to our hospital. However, since both Departments of Cardiology and Radiology are well known for their expertise in cardiac imaging, patients were referred to our University hospital from both colleagues in proximity to the clinic and colleagues in the greater Munich area.

In conclusion, we demonstrate that chemerin is strongly associated with markers of inflammation and components of the metabolic syndrome. Chemerin, however, does not predict coronary atherosclerotic plaque burden or morphology.

\section{Declaration of interest}

The authors declare that there is no conflict of interest that could be perceived as prejudicing the impartiality of the research reported.

\section{Funding}

This research did not receive any specific grant from any funding agency in the public, commercial, or not-for-profit sector.

\section{Acknowledgements}

We are indebted to Elisabeth Fleischer-Brielmaier, Inge BillerFriedmann, and Kerstin Henze for expert technical assistance.

\section{References}

1 Rajala MW \& Scherer PE. Minireview: the adipocyte - at the crossroads of energy homeostasis, inflammation, and atherosclerosis. Endocrinology 2003144 3765-3773.

2 Wittamer V, Franssen JD, Vulcano M, Mirjolet JF, Le Poul E, Migeotte I, Brézillon S, Tyldesley R, Blanpain C, Detheux M, Mantovani A, Sozzani S, Vassart G, Parmentier M \& Communi D. Specific recruitment of antigen-presenting cells by chemerin, a novel processed ligand from human inflammatory fluids. Journal of Experimental Medicine 2003198 977-985.

3 Bozaoglu K, Bolton K, McMillan J, Zimmet P, Jowett J, Collier G, Walder K \& Segal D. Chemerin is a novel adipokine associated with obesity and metabolic syndrome. Endocrinology $2007 \mathbf{1 4 8}$ 4687-4694.

4 Goralski KB, McCarthy TC, Hanniman EA, Zabel BA, Butcher EC, Parlee SD, Muruganandan S \& Sinal CJ. Chemerin, a novel adipokine that regulates adipogenesis and adipocyte metabolism. Journal of Biological Chemistry 2007282 28175-28188.

5 Cash JL, Hart R, Russ A, Dixon JP, Colledge WH, Doran J, Hendrick AG, Carlton MB \& Greaves DR. Synthetic chemerinderived peptides suppress inflammation through ChemR23. Journal of Experimental Medicine 2008205 767-775.

6 Barnea G, Strapps W, Herrada G, Berman Y, Ong J, Kloss B, Axel R \& Lee KJ. From the cover: the genetic design of signaling cascades to record receptor activation. PNAS 2008105 64-69.

7 Zabel BA, Nakae S, Zúñiga L, Kim JY, Ohyama T, Alt C, Pan J, Suto H, Soler D, Allen SJ, Handel TM, Song CH, Galli SJ \& Butcher EC. Mast cell-expressed orphan receptor CCRL2 binds 
chemerin and is required for optimal induction of IgE-mediated passive cutaneous anaphylaxis. Journal of Experimental Medicine 2008205 2207-2220.

8 Yoshimura T \& Oppenheim JJ. Chemerin reveals its chimeric nature. Journal of Experimental Medicine 2008205 2187-2190.

9 Wittamer V, Bondue B, Guillabert A, Vassart G, Parmentier M \& Communi D. Neutrophil-mediated maturation of chemerin: a link between innate and adaptive immunity. Journal of Immunology $2005175487-493$.

10 Takahashi M, Takahashi Y, Takahashi K, Zolotaryov FN, Hong KS, Kitazawa R, Iida K, Okimura Y, Kaji H, Kitazawa S, Kasuga M \& Chihara K. Chemerin enhances insulin signaling and potentiates insulin-stimulated glucose uptake in 3T3-L1 adipocytes. FEBS Letters $2008 \mathbf{5 8 2} 573-578$.

11 Broedl UC, Lebherz C, Lehrke M, Stark R, Greif M, Becker A, von Ziegler F, Tittus J, Reiser M, Becker C, Göke B, Parhofer KG \& Leber AW. Low adiponectin levels are an independent predictor of mixed and non-calcified coronary atherosclerotic plaques. PLoS ONE 20094 e4733.

12 Greif M, Becker A, von Ziegler F, Lebherz C, Lehrke M, Broedl U, Tittus J, Parhofer K, Becker C, Reiser M, Knez A \& Leber AW. Pericardial adipose tissue determined by dual source CT is a risk factor for coronary atherosclerosis. Arteriosclerosis, Thrombosis, and Vascular Biology 200929 781-786.
13 Tedgui A \& Mallat Z. Cytokines in atherosclerosis: pathogenic and regulatory pathways. Physiological Reviews 200686 515-581.

14 Gualillo O, González-Juanatey JR \& Lago F. The emerging role of adipokines as mediators of cardiovascular function: physiologic and clinical perspectives. Trends in Cardiovascular Medicine 2007 $17275-283$.

15 Reilly MP, Iqbal N, Schutta M, Wolfe ML, Scally M, Localio AR, Rader DJ \& Kimmel SE. Plasma leptin levels are associated with coronary atherosclerosis in type 2 diabetes. Journal of Clinical Endocrinology and Metabolism 200489 3872-3878.

16 Reilly MP, Lehrke M, Wolfe ML, Rohatgi A, Lazar MA \& Rader DJ. Resistin is an inflammatory marker of atherosclerosis in humans. Circulation $2005111932-939$.

17 Haffner SM. The metabolic syndrome: inflammation, diabetes mellitus, and cardiovascular disease. American Journal of Cardiology 200697 3A-11A.

18 Ridker PM, Hennekens CH, Buring JE \& Rifai N. C-reactive protein and other markers of inflammation in the prediction of cardiovascular disease in women. New England Journal of Medicine $2000342836-843$.

Received 8 May 2009

Accepted 1 June 2009 